\title{
Throughput Analysis Considering Capture Effect in IEEE 802.11 Networks*
}

\author{
Ge Xiaohu, Yan Dong, and Zhu Yaoting \\ Dept. Electronics \& Information Engineering \\ Huazhong University of Science \& Technology, Wuhan, P.R. China \\ \{xhge, dongyan, ytzhu\} @mail.hust. edu.cn
}

\begin{abstract}
The impact of capture effect on the IEEE 802.11 networks with different transmission speeds is investigated in this paper. A new Markov chain model considering capture effect for the binary exponential back-off scheme in the MAC layer has been proposed in the first time. Based on the new Markov chain model, a new throughput model is proposed, and then the impact of capture effect on throughput has been analyzed in the condition of different transmission speeds. The performance analysis shows that, in the RTS/CTS scheme, the improvement throughput of high speed networks caused by capture effect is more than that of low speed networks.
\end{abstract}

Keywords: 802.11 protocols, MAC layer, Markov chain, capture effect.

\section{Introduction}

Accompanying the standardization and rapid deployment of IEEE 802.11 Wireless Local Area Networks (WLANs) in the new century [1]. The medium access control (MAC) protocol used in IEEE 802.11 WLANs is called distributed coordination function $(\mathrm{DCF})$. Unsuccessful packets will be retransmitted according to a binary exponential back-off (BEB) policy.

For wireless communications, when multiple mobile stations send their data packets simultaneously to the same access point, a packet collision occurs. But one of the collided packets can still be correctly received, if its SINR seen by the access point is high enough. This phenomenon is called the capture effect [2]. The throughput performance of IEEE 802.11 WLANs has been extensively studied in literatures $[3,4]$. These previous related work does not consider the impact of capture effect on the IEEE 802.11 BEB back-off policy in the modeling and analysis of throughput performance. In contrast, we will consider this impact in this paper. Incorporating capture effect and physical layer parameters, a new Markov chain based access model will be used to analyze the throughput performance of IEEE 802.11 WLANs in the different transmission speeds.

The rest of the paper is organized as follows. In section II, a new throughput model based on the new Markov chain model is proposed, and then the improvement percent

* Supported by National Science Foundation of China under Grant No. 60610106111. 
of throughput caused by capture effect has been reported in condition of the different transmission rates. Finally, in section III, we conclude the paper and introduce some subjects of future research.

\section{Performance Analysis in Different Transmission Speeds}

\subsection{Throughput Model}

To analyze the MAC-layer performance of a WLAN BSS, we can build a discrete Markov chain model to characterize the temporal evolution of each node's status at the instants of state changes. In contrast to the previous related work, which does not consider the relationship between capture and transmission probabilities in the mathematical modelling, we design a new Markov chain model with capture effect and derive the closed-form analytical solutions [5].

Based on the Markov chain model derived from [5], the normalized throughput of AP can be calculated as the ratio of time occupied by the transmitted information to the interval between two consecutive transmissions

$$
S=\frac{P_{S} P_{t r} E[P]}{\left(1-P_{t r}\right) \sigma+P_{t r} P_{s} T_{s}+P_{t r}\left(1-P_{s}\right) T_{f}}
$$

where $E[P]$ is the average packet payload size; $P_{t r}$ is the probability that there are at least one transmission in the interval; $P_{S}$ is the conditional successful transmission probability; $P_{t r} P_{s}$ is the probability of a successful transmission occurred in the interval, and the average amount of payload information successful transmitted in the interval is $P_{t r} P_{s} E[P]$. The average length of the interval is readily obtained considering that, with probability $1-P_{t r}$, the interval is empty; with probability $P_{t r}\left(1-P_{s}\right)$ it contains a failure transmission. $T_{s}$ is the average time the channel is sensed busy because of a successful transmission, and $T_{f}$ is the average time the channel is sensed busy by a failure transmission, and $\sigma$ is the duration of an empty slot time. For simplicity, we assume that all nodes in this network have a fixed frame size and transmission rate, in order to keep $E[P], T_{s}, T_{f}$ and $\sigma$ constant.

And then, we can get a new expression of throughput $S$ considering capture effect from (1). In order to investigate capture effect into the IEEE 802.11 networks with different transmission speeds (1Mbit/s and 11Mbit/s), based on the RTS/CTS access scheme, the standard parameters of IEEE $802.11 \mathrm{~b}$ networks have been used in the following simulations.

\subsection{Performance Analysis Capture Effect in Different Transmission Speeds}

In the low speed networks (such as 1Mbit/s transmission speed), Fig. 1(a) shows the improvement percent of throughput considering capture effect, which is compared with throughput no considering capture effect. And this improvement percent of 


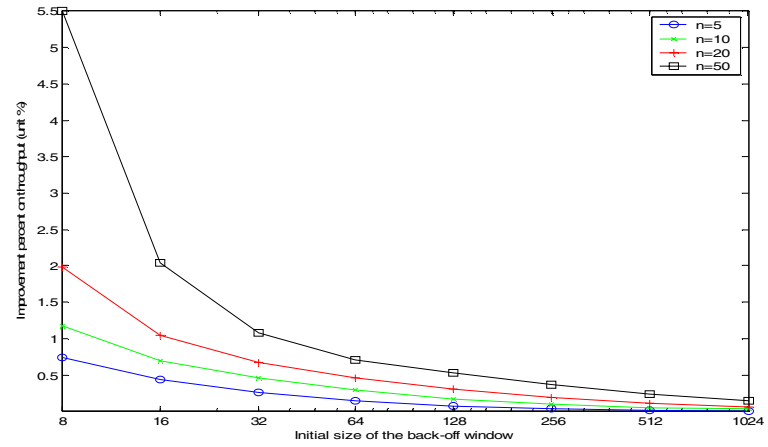

(a)

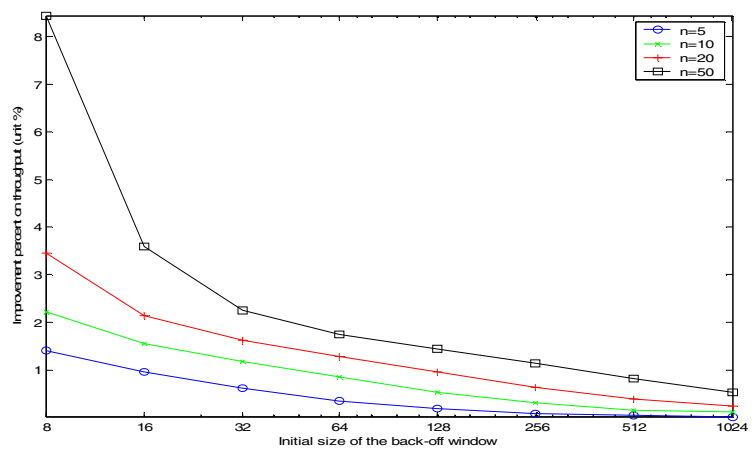

(b)

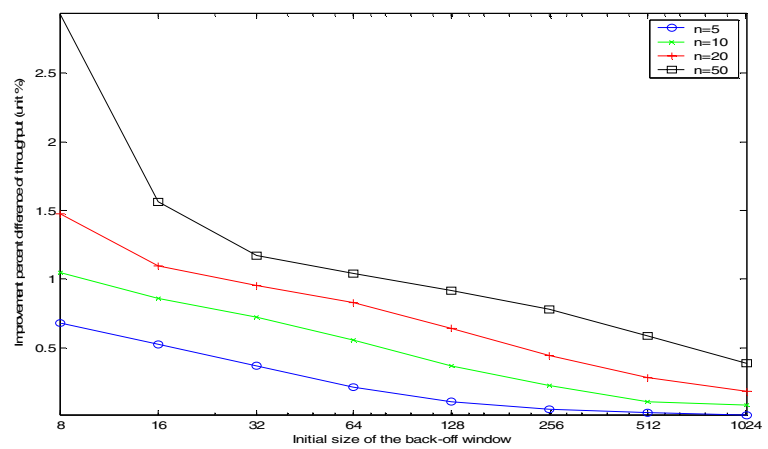

(c)

Fig. 1. Throughput analysis of 802.11 networks in different speeds

throughput has been plotted as the function of initial size of back-off window. It can be found that the improvement percent of throughput caused by capture effect is decreased, while the initial size of back-off window is increased. In the different network scales, the improvement percent of throughput is different, which is increased with the size of network. So the impact of capture effect in the low speed 
networks is increased with the size of network, but is decreased with the initial size of back-off window. The similar results can be obtained in the high speed networks (such as 11Mbit/s transmission speed), which can be shown in the Fig. 1(b).

In order to investigate the impact of capture effect into different transmission speeds, we compare the improvement percent difference of throughput between the high speed networks and low speed networks. The improvement percent difference of throughput, which means that the values of improvement percent in the Fig. 1(a) minus the values of improvement percent in the Fig. 1(b), is obtained and plotted in the Fig. 1(c). Fig. 1(c) shows that capture effect has more influence on the high speed networks than that on the low speed networks.

\section{Conclusion}

In this paper, a new IEEE 802.11b throughput model has been proposed based on the new Markov chain model [5]. By means of analysis of improvement throughput in the RTS/CTS access scheme, it can be found that the improvement percent of throughput in high speed networks is more than that in low speed networks, which means that capture effect has more influence on the high speed networks that that on the low speed networks in the aspect of throughput.

\section{References}

1. B. P. Crow, I. Widjaja, J. G. Kim, and P. T. Sakai. "IEEE 802.11 wireless local area networks," IEEE communications magazine, vol.35, Sept. 1997, pp.116-126

2. Z. Hadzi-Velkov and B. Spasenovski, "Capture Effect with Diversity in IEEE $802.11 \mathrm{~b}$ DCF”, IEEE International Symposium on ISCC 2003, vol.2, 30 June-3 July 2003, pp699 704

3. G. Bianchi, "Performance analysis of the IEEE 802.11 distributed coordination function," IEEE J.Selected Areas in Communications. Vol.18, Mar.2000, pp535-547

4. S. T. Sheu and T. F. Sheu, "A bandwidth allocation/sharing/extension protocol for multimedia over IEEE 802.11 ad hoc wireless LANs," IEEE J.Selected Areas in Communications, Vol.19, Oct.2001, pp2065-2080

5. X. Ge, Y. Dong, Y. Zhu, "Throughput Model of IEEE 802.11 Networks with Capture Effect,” IEEE WiCom, 2006. Wuhan, China, 22-24 Sept. 2006. 